Int. J. of Electronics Engineering and Applications, Vol. 7, NNo. 2, 2019

\title{
REGRESSION BASED SUB - IMAGE MATCHING METHODOLOGY FOR RECOGNIZING AN INDIAN PAPER BILL WITH A PARTIALLY CAPTURED BILL IMAGE
}

\author{
Amarnath R and Dr. P. Nagabhushan
}

\begin{abstract}
This paper aims to create visibility for visually impaired people or atleast to create ability for them to get the details of content which could be visible to the normal pair of eyes. System enables visually impaired users to leverage the camera of a mobile device to accurately recognize currency bills even if the images are partially or highly distorted. Once the face value of the currency bill is recognized, the mobile phone announces the denomination value of the bill. A captured sub image is matched across the definite set of bills in the knowledge base. The proposed method was experimented with manually created database containing 56 denominations of both newer and older bills for the knowledgebase or training. Moreover 560 randomly chosen partial imag es are acquired from all the denominations, which also include newer as well as older bills. The overall accuracy rate achieves $87 \%$ when all the bills are taken into consideration. $100 \%$ accuracy rate was achieved when using only newer bills for comparison.
\end{abstract}

Index Terms visually impaired people, recognize currency bills, the mobile phone, partial images, accuracy rate.

Reference to this paper should be made as follows: Amarnath R and Dr. P. Nagabhushan, (2019), "Regression Based Sub - Image Matching Methodology For Recognizing An Indian Paper Bill With A Partially Captured Bill Image" Int. J. Electronics Engineering and Applications, Vol. 7, No. 2, pp. 10-23.

\section{Biographicalnotes:}

Amarnath $\mathbf{R}$ completed his bachelors in Computer Science and Engineering from Anna Univeristy (Coimbatore), in 2010 and Masters in Computer Cognition and Technology from University of Mysore, India, during 2013.

Soon after his bachelor, he has served in a product based company named Amphisoft Technologies Private Limited formore than a period of 2 years. After his masters, he rejoined his previous employer in Research \& Development division, Amphisoft Technologies. His areas of interest include Image Processing, Video Processing, Virtual Reality, Pattern Recognition, Web, Mobile and Related Technologies.

Dr. P. Nagabhushan completed his bachelors in Electrical Engineering from SJCE Mysore, India and Masters from BITS Pilani, India. He obtained his Ph.D. from University y of Mysore, India. He has over 300 publications in reputed journals and conferences. He is an active researcher in the areas pertaining to Pattern Recognition, Image and Video Processing, Symbolic Data Analysis and Computational Intelligence. He is a Fellow of Institution of Engineers. 


\section{INTRODUCTION}

The most important sensory organ is the pair of eyes through which brain receives a large volume of inputs. However, these inputs are based on the visibility property. Even the 20/20 normal human eyes fail to experience this visibility in certain cases such as darkness or fog conditions. Indeed, it is difficult to imagine the situations with the visually impaired folk. Many practical difficulties are encountered by them every day. Some of the difficulties are reading the characters on the bus plates, identifying the routes, reading books etc. However, some of the obstacles encountered by visually impaired people can potentially be overcome through simple and cheap assistive software which runs on modern mobile phones.

Visually impaired individuals are increasing in number in the present world. The world's largest number of sightless people belongs to India. Over 15 million people out of the 37 million blind people of the world are Indians [15]. However, the technology is not always designed with this group of people in mind. Better electronic accessibility tools to fulfill their daily needs are developed and such assistive tools are expensive and specialized. Sometimes there are heavy devices which visually impaired individuals had to independently look after and carry around.

Despite the rapidly increasing use of credit cards and other electronic forms of payment, cash is still widely used for everyday transactions due to its convenience, perceived security and anonymity. The ability to correctly and

effortlessly exchange cash is fundamental for our everyday needs. Many simple transactions which are conducted each day, such as buying groceries or dining at a restaurant, require not only the handing over of paper bills but, more often than not, the return of some change. Unfortunately, in certain countries, visually impaired individuals cannot distinguish banknotes of different denominations from one another.

The past few years have witnessed an exponential growth in the computing capabilities and onboard sensing capabilities of mobile phones. Thus the mobile phones are an impeccable tool for building powerful and varied applications. Many applications could be developed and packaged into mobile phones.

One such practical application is taken into consideration is to recognize an Indian Bill Image which is captured using a mobile phone with an inbuilt miniature camera.

\section{A. Challenges Using Currency Bills}

United States dollar bills of different denominations all have the same size and so cannot be easily separated by measuring or comparing one against another. To make things worse, there are no tactile markings or other forms of identification on each bill to enable a blind person to discover its value without any sighted assistance. In 120 other countries, including the countries which have adopted the Euro, paper currency are of different sizes, [15]. However, in the European Union, despite the fact that Euro bills of larger denominations have larger sizes, it might still not be easy to separate them quickly and effortlessly. The difference between the sizes of these Euro bills might not be as drastic as some individuals would have required. The difference in width between a 5 Euro bill, for example, which is the smallest in denomination, and a 500 Euro bill, which is the largest, is only 2 centimeters. Indian paper bills denominations are of different sizes and intaglio has been introduced on the left of the watermark window. But this special mark fades out after a circulation of the bill. Thus it becomes an overhead for the visually impaired to recognize accurately. 


\section{B. Algorithmic Challenges Using Currency Bills}

Identifying banknotes using mobile phone software can be, therefore, algorithmically challenging as such software requires a high accuracy and speed. When a blind user wants to find out the value of a particular piece of currency, he or she will not be aware of the intensity or even of the availability of a suitable light source. So, the software should work under various and even under poor lighting conditions. Similarly, one cannot expect a visually impaired individual to place the banknote straight in front of the phone's camera, removing any other objects, such as furniture, which might happen to be in the background. No assumptions could be made about which face of the bill would be pointed towards the camera, or that a specific background, such as a white-colored table surface, would always be used.

A blind person might not even know the exact location of the camera's lens and so might inadvertently position a finger or another protrusion in between the phone and the banknote. It would be an inconvenience for the user to have to unfold each banknote and position it in a very specific orientation or with a specific face pointing towards the phone. In fact, the user would most probably present banknotes to the system which would be folded, rotated with various angles and at various distances from the camera's lens. Pictures taken by a visually impaired user might be blurred. This is because, the quality of the pictures gathered from mobile devices can be highly variable. Some images may be so blurred that even human recognition would be hard. In summary, a successful currency recognition algorithm needs to work accurately under various lighting conditions and even when bills are folded or covered by fingers, i.e. under partial occlusion, whilst banknotes need to be identified from several angles and distances.

First section started with introduction , followed by Algorithmic Challenges. Section 2, explains State of Art comprises of Literature Survey and Software Packages. Section 3 focuses on proposed algorithm and strategies in detail. Experimental analysis with apriori and posteriori is widely discussed in Section 4. Final Section describes the conclusion with future enhancements.

\section{RELATED WORK}

In prior work [1- 6], the authors have attempted to use very specific and specialized techniques when tackling the problem of recognising a paper currency bill.

Indian Currency Note Denomination Recognition using Color Images was proposed in [1]. In this work, recognition system is specific to the color of the Indian currency bill. This system is certainly dependent on the color factors of image bills obtained by the camera. Even though this system works on both faces of the bill, it seems that it still requires the particular face to be exposed fully to the camera and so would not work on folded bills or partial bills. However, the system may also fail due to rotation, distortion, scaling and dirtied (soiled) images. Again, the color factor may also undergo changes during image acquisition by the camera. It may vary during different lighting conditions and illumination changes.

A Fast Mobile Money Reader for the Blind was proposed in [2]. The authors have used Scale Invariant Feature Transform (SIFT) descriptors to recognize the currency bills. The SIFT algorithm identifies key-points or descriptors within any image and generates a multi-dimensional feature vector representation of each key-point. SIFT has been used to perform robust classification in the face of positional, rotational and scale variants. However, the system may fail due to its time consuming process to extract the key-points from the images (video streams) and comparing the keypoint features with one another. Moreover, the system may not work under illumination and lighting 
changes, since the algorithm uses only gray band of the captured images. The system requires a minimum of 60 to 70 percentage of the bill to be exposed to the camera.

Blind-Folded Recognition of Bank Notes on the Mobile Phone in [3] proposes the method to recognize the currency bill. The authors have attempted to make use of OCR (Optical Character Recognition) to recognize the face value of the bill. However, the system may not work while the image captured does not contain the face value of the bill. The system may not work properly with the skewed or rotated bill images. Therefore, the image acquisition is dependent on the orientation, alignment factors of the bill images.

Authors in [4][5] have proposed A Camera Phone Based Currency Reader for the Visually Impaired. As in [3], the authors have also attempted to make use of OCR to recognize the face value of the bill. The system is independent of rotation or skewed images. And moreover, the system works well with the partially captured image. However, the system may not work when the captured image does not contain the face value.

The AdaBoost learning algorithm was used in [5], to train a set of weak classifiers in order to determine the value of dollar bills. These weak classifiers consisted of a set of specific pixel pairs on each bill which were picked by the researchers. However, even though this system works on both faces of the bill, it seems that it still requires the particular face to be exposed fully to the camera and so would not work on folded bills or bills with distortion. The blind user would still need to orient each bill and take care where the phone's camera was being pointed, a procedure hard to perform when standing in line at a store. In comparison to a robust and well established image recognition algorithm which can reliably extract distinguishing features from any image presented, the authors' procedure of selecting numerous distinct pixel pairs for each bill cannot easily scale, especially when having to port the system to more currencies of other countries.

Similarly, in [6], specific regions which contain "characteristic saliencies that differ enough from one bill to the other" were selected. These characteristic image regions were also rotated in order to create more training samples which were then fed to an image recognition algorithm. However, although identifying bills under multiple orientations is very useful to a visually impaired individual, the distinguishing regions for each bill are still picked by hand and do not cover the whole of the bill's surface.

The idea of finding specific unique characteristics which could easily separate one banknote from another was taken a step further in [13]. The authors employed an algorithm which would help the user move the bill until the denomination's value printed on it would be exposed to the camera. Asking the user to move the banknote around until a region of interest has been located, however, can be tedious and time consuming.

Using real humans as a part of the image recognition process was proposed in [14]. In this work, images are first labeled by using an automatic approach such as a database or an image search engine and the results are validated in a distributed manner by paid human workers through Amazon Mechanical Turk. This system can certainly be adapted and used by the visually impaired to identify their banknotes. However, the use of human workers means that the users would need to incur at least some financial cost, a fact which can be avoided by building a completely automatic and highaccuracy currency recognition system which could also run without any connection to an online service.

Most of the work proposed in the literature, use the entire currency bills. It is ideally difficult for the visually impaired to capture the entire bill with correct orientation or alignment. Even though some literature [18] uses partial currency bills, still it requires face value to be exposed to the camera. Most of the literature is dependent on the simple background during image acquisition of bill. One such 
proposed paper, had worked on currency bill recognition system with partially captured image irrespective of face value on the images. The system requires immense processing power and time consuming henceforth. Still smart phones are not so capable enough to handle or manage the process of a huge video stream (images).

Most of the proposed work is dependent on rotation, scaling, illumination and lighting conditions. Few were attempted to work on shredded, torn, dirtied images but their accuracy rate in recognition is still challenging.

\section{PROPOSED WORK}

This section explains various methodologies and algorithms used to recognize partially captured currency bills.

Image Acquisition

The image is taken from a mobile camera and is stored in a 3D array. RGB (Red-Green-Blue) is converted into Gray Scale Image, which is represented in 2D array. The coordinates of the pixel in 2D image are given by the first and second index of the array and the third index stores the RGB intensities for each coordinate. Each element of array stores an unsigned 8 bit integer (0-255). The limit of first two indices of array determines the resolution of the image. In the current scenario, this limit is set to be 640 for the first index and 480 for the second index.

Due to its high processing time, the resolution of the image is reduced to $640 * 480$, which is considered to be optimal for the system environment. The Gray Scale Image is to be stored in JPEG format.

The images are taken under the following assumptions:

The knowledge base images are taken under proper lighting conditions.

No occlusion or shadowing is allowed in the image which is taken in a clear environment.

Distance of camera is nearly fixed from the object and within a small range of variation.

The orientation of the currency notes was such that the sufficient amount of data required for further processing of even a single face was at least visible.

The currency notes are of good quality i.e. they are not very much full of stains or dust etc. 


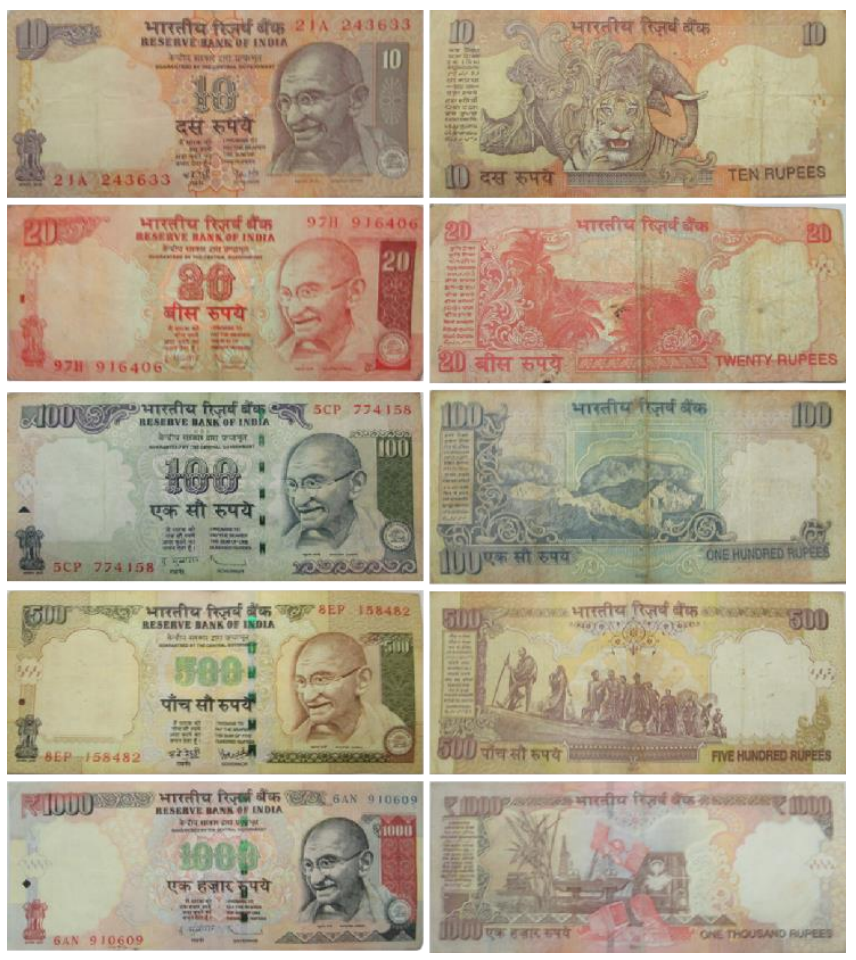

Fig. 1. Sample set of Indian Bank Bills in Knowledge Base with same scale, lighting, orientation. Sequential Searching with a Partial Query Image using Cumulative Histogram and Regression Line This section presents how a partial image is matched with the knowledge base (KB) image(s). In this case, two more assumptions are taken into consideration. Firstly, working with 3 layers namely Red, Green and Blue is time consuming and so the RGB images of both testing and training images are converted into gray scale images. Secondly, the acquired testing image is considered to have same scale with that of the knowledge base image. Thirdly, the testing image size must have at least 200 pixels, where experiment has been conducted to fix a minimum testing image size. Threshold value 200 is fixed based on the training database images, comparing it with partially captured images. Finally, it is assumed that the testing image should possess same lighting and orientation with that of $\mathrm{KB}$ images. With these criteria an algorithm is proposed. As far as matching is concerned, in the literature there are various pattern matching techniques, the background subtraction is the most commonly used technique. But, background subtraction algorithm fails drastically when there is a slight deviation (even at the degree of 1) in the angle. In the proposed algorithm, a novel histogram distance matching is used. A histogram distance measure based on cumulative histogram \& regression had been proposed by Nagabhushan and Pande [16]. Simplification of the formulation of this distance measure by refining the 4 cases into just 2 cases which is proposed by Nagabhushan and Pradeep Kumar [10] is likely to be followed in algorithm.

\section{ALGORITHM: SUB IMAGE MATCHING - SEQUENTIAL SEARCHING ALGORITHM}

Input: A query image (QI - Partial note) and KB images

Output: Recognition of a query sub-image from KB images

Convert QI to normalized cumulative histogram and regression line.

Get the Size of QI, say n*m and size of KB image is $r^{*} c$. 
For each KB image, do the following

$\mathrm{n} * \mathrm{~m}$ mask traverses through $\mathrm{r}^{*} \mathrm{c}$, do the following

Get each patch $(\mathrm{P})$ of size $\mathrm{n} * \mathrm{~m}$ from the $\mathrm{KB}$ and convert $\mathrm{P}$ to cumulative histogram and regression line.

Compute area which covers the two regression line, namely the QI regression line and the patch $(\mathrm{P})$ regression line and also compute the distance between two TI and P.

Store area and distance value with $\mathrm{KB}$ denomination value in a vector (V).

End Loop

Find the minimum value in $\mathrm{V}$ and fetch the $\mathrm{KB}$ denomination value from the vector $\mathrm{V}$.

Results and Discussions

The algorithm is a sequential searching of a partial image from the knowledge image and also tracks a minimum distance out of all the denomination, which is diagrammatically shown in the figure 2,3 .
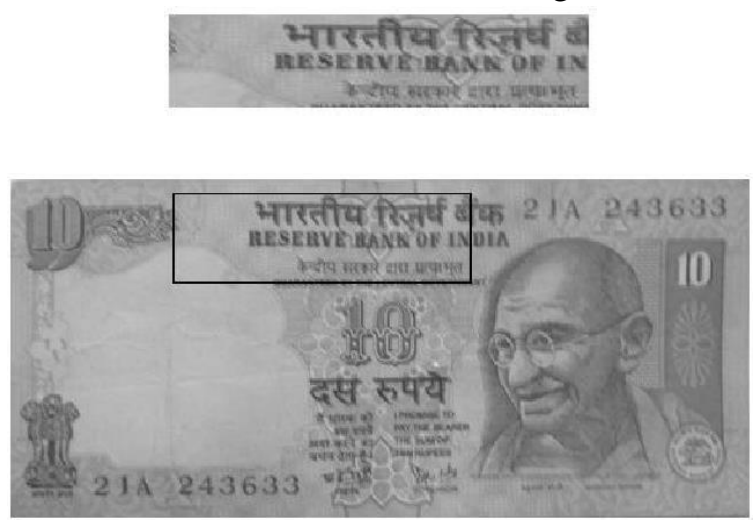

Fig. 2. Sample Result 1: Image at the top is a partial query image of denomination Rs.10. and the image at the bottom is matched denomination in the Knowledge Base (KB).
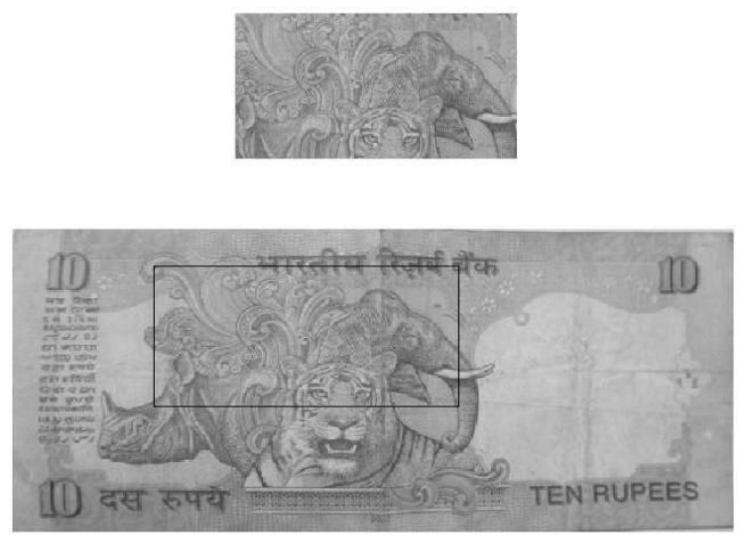

Fig. 3. Sample Result 2: Image at the top is a partial query image of denomination Rs.10. and the image at the bottom is matched denomination in the Knowledge Base $(\mathrm{KB})$.

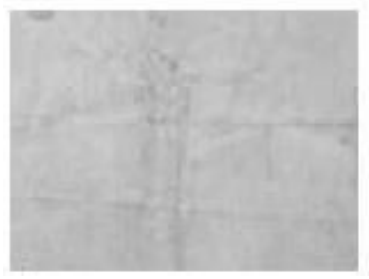


SUB IMAGE MATCHING - SEQUENTIAL SEARCHING ALGORITHM

The complexity of this a lgorithm is described as in a sequence of steps.

Step 1: Compute normalized cumulative histogram takes $\mathrm{O}(\mathrm{h} * \mathrm{w})$ and to compute regression line takes $\mathrm{O}$ $(\mathrm{h} * \mathrm{w})$. So it could be written as $\mathrm{O}(\mathrm{h} * \mathrm{w})+\mathrm{O}(\mathrm{h} * \mathrm{w})$, which could be rewritten as $\mathrm{O}(\mathrm{h} * \mathrm{w})$.

Step 2: PI traverses through $\mathrm{KI} * \mathrm{n}$.

This complexity is defined as $\mathrm{O}(((\mathrm{r}-\mathrm{h}) *(\mathrm{c}-\mathrm{w})) * \mathrm{n})$.

Step 3: Find the minimum in a vector is a ga in is defined a $\mathrm{O}(((\mathrm{r}-\mathrm{h}) *(\mathrm{c}-\mathrm{w})) * \mathrm{n})$.

The overall worst ca se complexity is defined as,

$\mathrm{T}_{\text {worst }}(1)=\mathrm{O}(\mathrm{h} * \mathrm{w})+\mathrm{O}(((\mathrm{r}-\mathrm{h}) *(\mathrm{c}-\mathrm{w})) * \mathrm{n})+\mathrm{O}(((\mathrm{r}-\mathrm{h}) *(\mathrm{c}-\mathrm{w})) * \mathrm{n})$

(1)

$$
=\mathrm{O}(\mathrm{h} * \mathrm{w})+\mathrm{O}(((\mathrm{r}-\mathrm{h}) *(\mathrm{c}-\mathrm{w})) * \mathrm{n})
$$

Supposen is considered to be 1 , then the worst case complexity from equation (2) is written as,

$\mathrm{T}_{\mathrm{worst}}(1)=\mathrm{O}(\mathrm{h} * \mathrm{w})$

But, in this case, $\mathrm{n}$ is not less than 14 , so worst case complexity is,

$\mathrm{T}_{\text {worst }}(1)=\mathrm{O}(((\mathrm{r}-\mathrm{h}) *(\mathrm{c}-\mathrm{w})) * \mathrm{n})$

Fig. 4. Query partial image with more knowledge base images are retrieved.

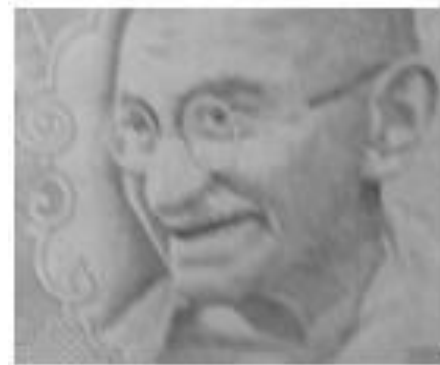

Fig. 5. Query partial image with more knowledge base images are retrieved

As the algorithm when tested with a single query Image, the matching criteria fail drastically in the situation where a common attribute lies in all the denominations. For an instance, the image patch showed in the fig. 4 which is a commonality on all the denomination. For another example, patch shown in the fig. 5 also lies on front face of all the denominations.

\section{EXPERIMENTAL ANALYSIS}

This section presents the experimental analysis, including how data have been collected for training and testing images of an Indian Currency bills. This section also describes various evaluation methodologies for testing with respect to the algorithm discussed in the previous section.

\section{A. Data Collection}

Data was collected by taking clear and complete pictures of

bills through the phone's camera under a uniform light source for knowledge base and on a white clear background. As literature is concerned, there is no any standard database available for currency 
bills. The phone camera held at same angle and distance for taking the sample KB images. The images were then pre-processed to remove the background so as to leave the currency image of the bill for further processing. Indian Rupee denomination consists of 14 images including front and back face of the denomination representing Rs. 5, 10, 20, 50, 100, 500 and 100. Each denomination consists of 2 faces. 14 images are collected which are clear new bills without any soil or dirt on it. Apart from those 14 images, 42 additional images are collected

which are considered to be old bills, because it is dirtied with 20 to 40 percent, due to circulation. Altogether 56 images are considered for KB. Every denomination carries 8 images, out of which 4 representing front and 4 for back face. Similarly, 560 sample partial query images are taken for testing. Out of which, 40 images of each face of the denominations are randomly chosen out from a complete image. Therefore a single denomination with respect to PR is trained and tested with 8 and 80 images. To be more specific, 8 images contains entire face of a denomination where as 80 images are of partial. Out of 80 partial images, 10 partial images are gathered from a new currency bills and remaining are of dirtied bills.

\section{B. Apriori Analysis}

In apriori analysis, only worst case scenario is taken into consideration. Size of a partial image (PI) is described as height $(\mathrm{h})$ and width (w). Size of a $\mathrm{KB}$ image $(\mathrm{KI})$ is described as row (r) and column (c). Assume that all the KB images are of same resolution with same height and width. The number of images in $\mathrm{KB}$ is represented as $\mathrm{n}$. Hand simulation is provided with respect to apriori analysis of Algorithm 1, with different scenarios. Let us assume that the dimension of a denomination in KB is almost fixed with a size of say $640 * 480$ and the dimension of a PI varies but not less than 196 pixels. Number of KI is multiple of 2. Minimum KI is defined with 14. In the first case, $n$ is kept constant with PI varying with 50 pixels.

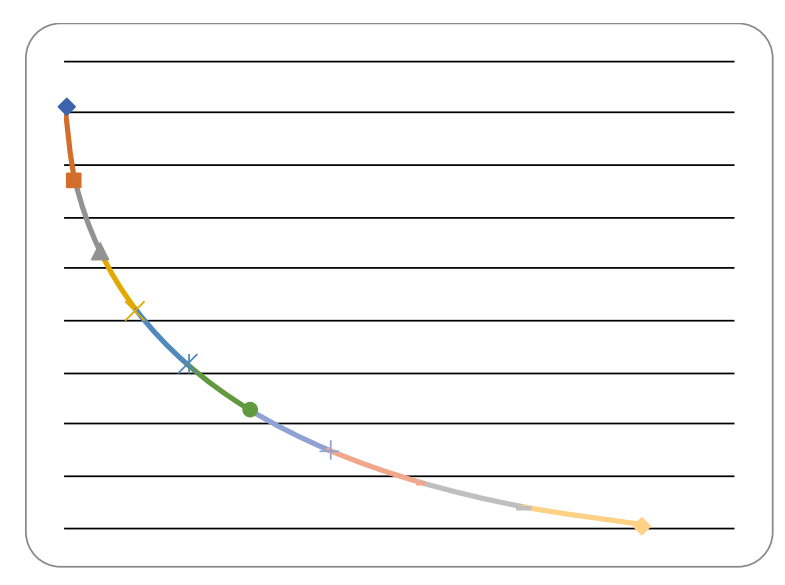

Fig. 6. Apriori analysis of algorithm with $\mathrm{n}$ is constant, PI varying with an addition of 50.

As the size of PI increase, the number of iterations reduces, which is depicted in the fig. 40. In the second case, PI is kept constant by which number of KI varies with the addition of 14 . 


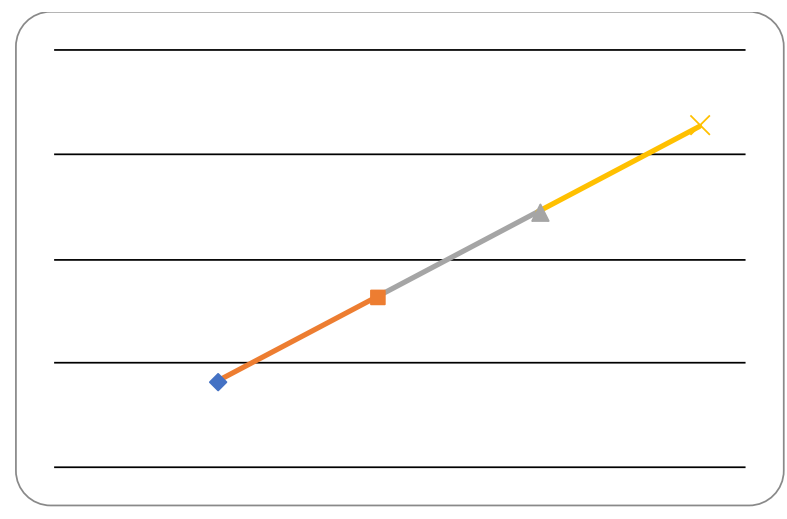

Fig. 7. Apriori analysis of algorithm with PI is constant and $n$ varying with an addition of 14 .

It is obvious that, fig. 41 depicts the linearity of the function by which $\mathrm{n}$ varying with an addition of 14. Since denomination is finite while considering the currency bill in $\mathrm{KB}$, so it is required that the same proportion must be added to each denomination in the KB.

\section{A. Posteriori Analysis}

Real time performance analysis is conducted by using the same data set which has been discussed in the previous section. In this section, two cases are considered. First case explores about the partial image varying with an addition of size 50 and remaining data are kept constant and second case explains the scenario where in the number of knowledge base images varies with an addition of 14 .

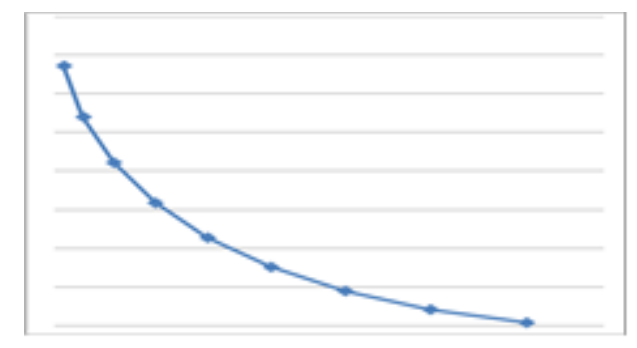

Fig. 8: Posteriori analysis of algorithm $n$ is constant, PI varying with an addition of 50.

Fig. 8 explores that the system works as same as that of apriori analysis. Since data of $\mathrm{x}$ and $\mathrm{y}$ axis are huge, it is certainly difficult to display on the graph shown in the fig. 43. As the dimension of partial image increases then the iteration decreases systematically.

Second scenario deals with the case, where in number of knowledge base images grow with an addition of 14 images respectively. As the previous section explains the linearity of the function with $\mathrm{n}$ in $\mathrm{KB}$ varies with an addition of 14 images which is reflected in the posterior analysis. Fig. 44 depicts the linearity function of the system. Since data labels of $x$ and $y$ axis are huge, they are not displayed in the figure 


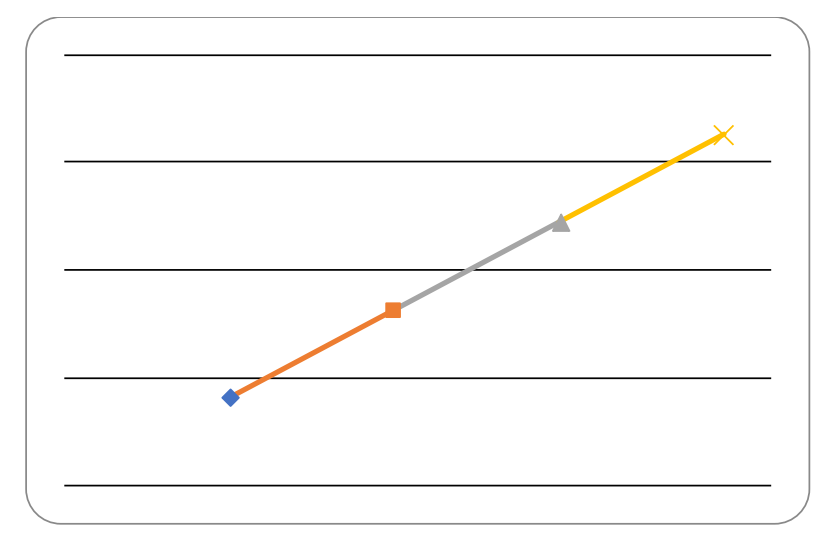

Fig. 9: Posteriori analysis of algorithm with PI is constant and $n$ varying with an addition of 14

The posterior analysis proves that the complexity of the algorithm is appropriate with the system developed and designed.

\section{EXPERIMENTAL RESULTS}

Experiment is conducted considering 6 scenarios. First case dealt with comparing new bills of PI with new KI bills. Second case presents comparing old bills of PI and KI. Third case discusses about comparing new bills of PI with old KI bills. Forth case is all about comparing old bills of PI with new KI bills. Fifth and final cases elaborate the overall comparison of new and old bills of training with new and old bills of testing. In each scenario, extensive experiment is performed by comparing 1 to $\mathrm{PI}$ images with 1 to $\mathrm{k} \mathrm{KI}$ images.

Out of $560 \mathrm{PI}$ and $56 \mathrm{KI}$, the overall accuracy rate carries to 90 percent.

Experimental results in the previous section shows the accuracy rate when it is compared with new currency bills contains 100 percent where as old bills reduces the overall accuracy rate. The moisture and noise on the bills significantly reduces the accuracy. This section detailed more about the error caused when matching is performed.

Table 14: Error Analysis

\begin{tabular}{|c|c|c|}
\hline 560 PI & Positive & Negative \\
\hline True & $477(86 \%)$ & $83(14 \%)$ \\
\hline
\end{tabular}

A negligible difference in capturing the partial image may vary significantly when comparison takes place. Moreover, the system uses only a single spectrum band, where it averages RGB values. Color factors of Rs. 20 and Rs. 1000 are more alike. The original color factors would vary when it is on circulation, so this may be the case, where in 14 percent is true negative. 


\section{CONCLUSION}

Vision to the invisible is the goal of this paper. Currency Recognition through mobile phone is challenging problem in the literature of PR and IP. Since mobile phone must possess the processing power required to compute the image as a whole. Image processing could be performed by the remote computer via Internet connection of the mobile phone. Mobile phone must be connected to the Internet, whenever it is needed, with sufficient data rate to send and receive across the Internet.

The images are acquired in a controlled environment, with suitable lighting conditions. With assumptions discussed in Section II, it is still a challenging part to achieve 100 percent accuracy. In particular, it is not tolerable to misclassify the bill, for example, 1000 rupees currency must not be recognised as 10 rupees bill. It is always required to achieve 100 percent accuracy for any data set used for testing. The overall accuracy is estimated as 90 percent when considering both new and old bills. While choosing only new bill data set, the proposed system achieves 100 percent accuracy. Most of the Indian currencies are dirtied due to circulation, so it mandatory to include the old bills along with the new bills in the dataset for testing

The system can be extended with the following ways:

1) To resolve, when a partially captured bill is considered to be a common attribute across all the denominations, shown in Fig 4 and 5.

2) Recognise rotational and scale invariant partially captured bill image. 


\section{REFERENCES}

[1 Hanish Aggarwal, Pa dam Kumar, "Indian Currency NoteDenomination Recognition in Color Images", International Journal on Advanced Computer Engineering and Communication Technology Vol-1 Issue: 1: ISSN2278-5140.

[2] Nektarios Paisios, Alexander Rubinsteyn, Lakshminarayanan Subramanian, "Exchanging Cash with no Fear: A Fast Mobile Money Reader for the Blind".

[3] Sta vros Papastavrou, Demetris Hadjiachilleos, Georgios Stylianou, "Blind-Folded Recognition of Bank Notes on the Mobile Phone",July 25-29, 2010. ISBN 978-1-4503-0210-4/10/0007.

[4] Xu Liu, "A Camera Phone Based Currency Reader for the Visually Impaired", ASSETS'08, October 13-15, 2008, Halifax, Nova Scotia, Canada, ACM 978-1-59593-976-0/08/10.

[5] X. Liu, D. Doermann, and H. Li. Mobile visual aid tools for users with visual impairments. Mobile Multimedia Processing, pages 21-36,2010.

[6] R. Parlouar, F. Dramas, M. Mac, and C. Jouffrais. Assistive device for the blind based on object recognition: an application to identify currency bills. In Proceed ings of the 11th international ACM SIGACCESS conference on Computers and accessibility, pages 227-228. ACM, 2009.

[7] H. Hassanpour and E. Hallajian, "Using Hidden Markov Models for Feature Extraction in Paper Currency Recognition".

[8] Chetan.B.V and Dr. P.A.Vijaya, "A Robust Side Invariant Technique of Indian Paper Currency Recognition”, International Journal of Engineering Research \& Technology (IJERT), ISSN: 2278-0181 , Vol. 1 Issue 3, May-2012.

[9] http://www.rbi.org.in/currency/banknotes.html, (accessed October 2012).

[10] R.Pradeep Kumar, P.Na gabhushan, "Multiresolution Knowledge Mining using Wavelet Transform ", Engineering Letters, 14:1,EL_14_1_30(Advance online publication: 12 February 2007).

[11] N. Paisios, A. Rubinsteyn, V. Vyas, and L. Subramanian. Recognising currency bills using a mobile phone: an a ssistive aid for the visually impaired. In Proceedings of the 24th annual ACM symposium adjunct on User interface software and technology, pages 19-20. ACM, 2011.

[12] N. Paisios, A. Rubinsteyn, and L. Subramanian. Exchanging cash with no fear: A fa stmobile money rea der for the blind. In Workshop on Frontiers in Accessibility for Pervasive Computing. ACM, 2012.

[13] S. Papastavrou, D. Hadjiachilleos, and G. Stylianou. Blind-folded recognition of bank noteson the mobile phone. In ACM SIGGRAPH 2010 Posters, page 68. ACM, 2010.

[14] T. Yan, V. Kumar, and D. Ga nesan. Crowdsearch: exploiting crowdsfor accuratereal-time image search on mobile phones. In Proceedings of the 8th international conference on Mobile systems, a pplications, and services, pages 77-90. ACM, 2010.

[15] http://npcb.nic.in/ (accessed October 2012).

[16] M.B. Sanjay Pande and P.Nagabhushan. An algorithmic model for exploratory analysis of trace elements in cognition and recognition of neurological disorders. Ph.D. Thesis. Department of studies in computer Science, University of Mysore. August, 2004.

[17] James D. Foley, Andries van Dam, Steven K. Feiner and John F. Hughes, Addison Wesley, Computer Graphics, 2005.

[18] Nektarios Paisios, Mobile Accessibility Tools for the Visually Impaired, PH.D. Thesis, Department of Computer Science, Courant Institute of Mathematical Sciences New York University.

[19] Amarnath R, Under the guidance of Dr. P. Nagabhushan, Major Project Report, Indian Paper Currency Bill Recognition System for the Visually Impaired, 2012-2013, M.Tech, Computer Cognition and Technology (CCT). 
[20] Anoop Joyti Sahoo, and Rajesh Kumar Tiwari "A Novel Approach for Hiding Secret data in Program Files" International Journal of Information and Computer Security. Volume 8 Issue 1, March2016,

[21] Abu Sa lim, Sachin Tripathi and Rajesh Kumar Tiwari "A secure and timestamp-based communication scheme for cloud environment” Published in International Journal of Electronic Security and Digital Forensics, Volume 6, Issue 4, 319-332.

[22] Rajesh Kumar Tiwari and G. Sahoo, "A Novel Watermark Scheme for Secure Relational Da tabases" Information Security Journal: A GlobalPerspective, Volume 22, Issue 3, July 2013. 